\title{
RECENT PROGRESS ON WARING'S THEOREM AND ITS GENERALIZATIONS*
}

\author{
BY L. E. DICKSON
}

1. Introduction and Summary. The simplest theorem in question states that every positive integer is a sum of four integral squares. This is an example of a universal Waring theorem. The elaborate theory due to Hardy and Littlewood yields a number $C(s, n)$ beyond which every integer is a sum of $s$ integral $n$th powers greater than or equal to zero. Since $C$ is excessively large, their theory yields essentially only asymptotic theorems.

For several years the writer has been elaborating his idea that it is possible to supplement these asymptotic theorems and show that they hold also for all integers below $C$. The resulting new universal theorems are here first published.

The various aspects of Waring's problem may be compared with those of the theory of functions of a complex variable. Such a function may be studied in the neighborhood of infinity (corresponding to asymptotic Waring theorems), or in the neighborhood of the origin (compare our new results in $\$ 2$ ), or over the whole plane (corresponding to universal Waring theorems). Analytic continuation of a function has its analog in our extension of a range for which $s n$th powers suffice to a larger range for which also $s n$th powers suffice. Such an extension is different from ascent to a still larger range for which $s+1 n$th powers suffice $(\$ 3)$.

2. On the Ideal Limit for the Universal Waring Theorem. It has been proved that every positive integer is a sum of nine cubes, and noted that 23 requires nine cubes ( 8,8 and seven 1 's), whence 9 is the ideal limit for cubes. For biquadrates (or fourth powers), 79, 159, 239, 319, 399, 479 and 559 require 19 biquadrates. The published table to 4100 shows that 19 biquadrates suffice to 4100 . But the best theorem to date is that all integers are sums of 35 biquadrates. Hence it is only on evidence by tables that 19 is called the ideal limit for biquadrates.

By a decomposition (into $n$th powers $\geqq 0$ ), we mean a linear

* An address delivered at Chicago on June 23, 1933, before the Society and Section A of the American Association for the Advancement of Science. 
homogeneous function of $1, a=2^{n}, b=3^{n}, c=4^{n}, \cdots$, with integral coefficients $\geqq 0$. The sum of the coefficients will be called the weight of the decomposition.

We shall prove results which indicate that the ideal limit for $n$th powers is $I=q+2^{n}-2$, where $q$ is the greatest integer $<(3 / 2)^{n}$. Thus

$$
b=q a+r, \quad(0<r<a) .
$$

Consider $P=q 2^{n}-1$. By (1), $P<3^{n}$, whence any decomposition of $P$ involves only 1 and $2^{n}$. But $P$ requires more than $I$ such terms except when there are exactly $q-1$ terms $2^{n}$ and exactly $2^{n}-1$ terms 1 . This proves that $P$ is a sum of $I$, but not fewer, $n$th powers.

Besides our results concerning $I$, which furnish a yard-stick for judging universal theorems, we shall derive various important theorems. In particular, after $P$ there is a rapid reduction below $I$ of the number of $n$th powers which suffice. This indicates that the known asymptotic results should be much improved in the future.

All integers $>P$ are sums of $T_{1} n$th powers, where $T_{1}$ is a certain integer $<I$. Beyond the last one requiring $T_{1}$, all are sums of $T_{2}<T_{1}$ powers, etc. Let $t$ denote the last $T_{i}<c$; this $t$ is the largest number (6) which is $<c$.

The following table gives the ideal $I$, this $t$, and expresses $t$ as a percent of $T$. Every integer not exceeding that in the last column is a sum of $I n$th powers.

\begin{tabular}{rrrcrrr}
$n$ & \multicolumn{1}{c}{$I$} & \multicolumn{1}{c}{$t$} & \multicolumn{1}{c}{$t / I$} & \multicolumn{3}{c}{$I$ suffices to } \\
\hline 7 & 143 & 97 & .68 & 26244 \\
8 & 279 & 152 & .54 & 446148 \\
9 & 558 & 333 & .60 & 4487724 \\
10 & 1079 & 523 & .48 & 17006112 \\
11 & 2132 & 1211 & .57 & 167226768 \\
12 & 4223 & 1523 & .36 & 485205633 \\
13 & 8384 & 2966 & .35 & 3124873080 \\
14 & 16673 & 5245 & .31 & 822670468 \\
15 & 33203 & 7481 & .23 & 21293780000
\end{tabular}

Define integers $Q$ and $R$ by the formulas 


$$
a=(a-r) Q+R, \quad(0 \leqq R<a-r) .
$$

Write $\lambda=(Q+R) b$ if $a \geqq q+r+R$, but $\lambda=(a+Q-q-r) b$ if $a<q+r+R$. We shall prove that every positive integer $<\lambda$ is a sum of $I$ integral $n$th powers $\geqq 0$.

The example $n=8$ will clarify the later theory. Evidently any integer can be expressed as the sum of a number $m a+k b$ and a number chosen from $0,1, \cdots, 255=a-1$. These sums are written in the last column of the following tablette:

$$
\begin{aligned}
& 66+(m+51) a+(k-2) b \quad 161+(m+25) a+(k-1) b\{m a+k b\} \\
& 161+ \\
& \{(m+26) a+(k-1) b\} \quad 95+ \\
& \{(m+52) a+(k-2) b\} \quad 95+\quad 190+
\end{aligned}
$$

Here the first (or second) row of dots takes the place of 94 lines obtained by adding $1,2, \cdots, 94$ in turn to the line above the dots, while for the third row of dots we add $1,2, \cdots, 65$. Hence there are altogether $95+95+66=256=a$ rows. The three numbers in any line are equal since $b=25 a+161$. The weight of a diagonal number in \{\} is less than the weights of the remaining two numbers of the same line. Similar results evidently follow for the rows indicated by dots.

Consider a fourth column obtained from the left one of our three columns by replacing a single $b$ by its value; then a fifth column obtained similarly from this fourth column; etc., until we reach a column free of $b$. It will be proved that the weight of every number in these added columns exceeds the weight of the number in \{\} in the same row and lying in our three columns. In other words, the weight of a number in \{\} in the three columns of our tablette is the minimum of the weights of all decompositions of an integer $(<c)$ in the same line. There remains only the simple comparison of these minimum weights for the various values of $m$ and $k$. The general theory makes this comparison for any $n$.

The three diagonal elements of our tablette are the values of $M_{0}, M_{1}, M_{2}$ in the following general theory. 
Lemma. Let $M_{s}=(m+s q+s) a+(k-s) b$, and $k \geqq x-1$. Then $j+M_{0}, \cdots, j+M_{x-1},(j=0, \cdots, a-r-1)$, together give decompositions of all integers $y+m a+k b$ for $y=0,1, \cdots, x(a-r)-1$.

By (1), $M_{s}$ is equal to $N_{s}=s(a-r)+m a+k b$. Also,

$$
a-r-1+N_{s}=N_{s+1}-1 .
$$

Hence the $j+N_{s}$ give all integers from $N_{0}$ to $N_{x}-1$ inclusive.

First, let $k<Q$. The $j+M$ in the lemma with $x=k$, together with $i+M_{k}$ for $i=0, \cdots, a-1-k(a-r)$, give decompositions of all the integers $z+m a+k b$ for $z=0, \cdots, a-1$. For the maximum $i$ the weight of $i+M_{k}$ is

$$
w_{m k}=a-1-k(a-r)+m+k q+k .
$$

For the maximum $j$, the weight of $j+M_{s},(0 \leqq s \leqq k-1)$, is $a-r-1+m+s q+k$. For the largest value $k-1$ of $s$, this weight will be $<w_{m}$ if $k(a-r)<q+r$. Since $k \leqq Q-1$, the latter follows from

$$
r-1=(a-r)(Q-1)+R-1,
$$

which is derived by subtracting $a-r+1$ from (2). Hence $w_{m k}$ is the largest weight of all our decompositions.

For $m=0, \cdots, q-1$, the largest $w_{m k}$ is

$$
C_{k}=a-2-k(a-r)+q+k q+k .
$$

For $m=q$, we shall employ $y+q a+k b$ only for $y=0, \cdots$, $r-1$, since $y=r$ would yield $(k+1) b$ by (1), which exceeds the integers considered in Theorem 1. Previously we had $y=0, \cdots$, $a-1>r-1$. We have therefore suppressed one or more of the high values of $i$ in $i+M_{k}$, and increased $m$ from $q-1$ to $q$. Hence the new greatest weight is $\leqq C_{k}$. This proves the following result.

Theorem 1. For $k<Q$ every integer $\geqq k b$ and $<(k+1) b$ has a decomposition whose weight is $\leqq C_{k}$.

Second, let $k \geqq Q$. By the lemma with $x=Q$ we see that

$$
\begin{array}{rr}
j+M_{0}, \cdots, j+M_{Q-1}, & (j=0, \cdots, a-r-1), \\
i+M_{Q}, & (i=0, \cdots, R-1),
\end{array}
$$


together give decompositions of $w+m a+k b$ for $w=0, \cdots$, $R-1+Q(a-r)=a-1$, by (2). The greatest weight is the larger of

$$
\begin{aligned}
& b_{m k}=a-r-1+m+(Q-1) q+k \\
& d_{m k}=R-1+m+Q q+k .
\end{aligned}
$$

For $m=0, \cdots, q-1$, the largest $b_{m k}$ or largest $d_{m k}$ is, respectively,

(6) $B_{k}=a-r-2+Q q+k, D_{k}=R-2+q+Q q+k$.

For $m=q$, we shall employ $z+q a+k b$ only for $z=0, \cdots$, $r-1$, since $z=r$ yields $(k+1) b$, which exceeds the integers considered in Theorem 2. In view of (3) and the Lemma for $x=Q-1$, we now employ

$$
\begin{array}{r}
j+M_{0}, \cdots, j+M_{Q-2}, \quad(j=0, \cdots, a-r-1), \\
i+M_{Q-1}, \quad(i=0, \cdots, R-1),
\end{array}
$$

which together give decompositions of $z+q a+k b,(z=0, \cdots$, $r-1)$. The greatest weight is the larger of

$$
\begin{gathered}
a-r-1+q+(Q-2) q+k<B_{k}, \\
R-1+q+(Q-1) q+k<D_{k} .
\end{gathered}
$$

Evidently $B_{k} \geqq D_{k}$ if and only if*

$$
a \geqq q+r+R \text {. }
$$

THEOREM 2. For $k \geqq Q$, every integer $\geqq k b$ and $<(k+1) b$ has $a$ decomposition whose weight is $\leqq L_{k}$, where $L_{k}$ is the larger of (6), whence $L_{k}=B_{k}$ if $a \geqq q+r+R$, and $L_{k}=D_{k}$ if $a<q+r+R$.

Employing (3), we see that

$$
C_{Q-1}=a-3+R-r+Q(q+1) .
$$

Thus $C_{Q-1} \geqq B_{k}$ if and only if $k \leqq R+Q-1$. Hence if $a \geqq q+r+R$ and $Q \leqq k \leqq R+Q-1$, Theorem 2 shows that every integer $\geqq k b$ and $<(k+1) b$ has a decomposition whose weight is $\leqq C_{Q-1}$. Apply this result for $k=Q, Q+1, \cdots, R+Q-1$ in turn. We obtain the following theorem.

* Except for $n=3,10,11,12$, (8) holds for $2 \leqq n \leqq 27$. Note that $Q=1$ (whence $R=r$ ) if and only if $a>2 r$. 
THEOREM 3. If $a \geqq q+r+R$, every integer $\geqq Q b$ and $<(Q+R) b$ has a decomposition whose weight is $\leqq C_{Q-1}$.

We shall omit the proof (by induction on $n$ ) that, in (1),

$$
a>q+r+1 \text {, }
$$

Hence $C_{k}$ decreases when $k$ increases, since

$$
C_{k-1}-C_{k}=a-q-r-1 \geqq 1 .
$$

We have $C_{0}=a+q-2=I, C_{1}=r+2 q-1, C_{2}=2 r-a+3 q$.

If an integer $h$ is a sum of $C n$th powers $\geqq 0$, we shall say that $C$ suffices for $h$. Evidently $G$ suffices for $h$ if $G>C$.

Theorem 4. For $k=0, \cdots, Q-1, C_{k}$ suffices for all integers $\geqq k b$ and $<Q b$.

This is true by Theorem 1 if $k=Q-1$. To proceed by induction from $k$ to $k-1$, let Theorem 4 hold for a certain $k$. Then $C_{k-1}$ (which exceeds $C_{k}$ ) suffices for integers $\geqq k b$ and $\angle Q b$. By Theorem $1, C_{k-1}$ suffices for integers $\geqq(k-1) b$ and $<k b$. The two results show that $C_{k-1}$ suffices for integers $\geqq(k-1) b$ and $<Q b$, whence Theorem 4 holds when $k$ is replaced by $k-1$.

Theorems 3 and 4 yield the following theorem.

TheOREM 5. Let $a \geqq q+r+R$. For $k=0, \cdots, Q-1, C_{k}$ suffices for all integers $\geqq k b$ and $<(Q+R) b$. In particular, $C_{0}=I$ suffices for all integers $<(Q+R) b$. If $k \geqq Q, B_{k}$ suffices for all integers $\geqq Q b$ and $<(k+1) b$.

The final statement follows from Theorem 2 .

Finally, let $a<q+r+R$. In Theorem 2, $L_{k}=D_{k}$. We shall have $C_{Q-1} \geqq D_{k}$ if $k \leqq a-1+Q-q-r$. Hence, as in the proof of Theorem 3 , every integer $\geqq Q b$ and $<(a+Q-q-r) b$ has a decomposition whose weight is $\leqq C_{Q-1}$. Combining this with Theorem 4 , we have the following additional theorem.

Theorem 6. Let $a<q+r+R$. For $k=0, \cdots, Q-1, C_{k}$ suffices for all integers $\geqq k b$ and $<(a+Q-q-r) b$. If $k \geqq Q, D_{k}$ suffices for all integers $\geqq Q b$ and $<(k+1) b$.

We shall define a tablette $T$ with $Q+1$ columns whose $a-1$ diagonal elements are (5) and which reduces to our special tablette when $n=8$. Let $s \leqq Q$. Write $D$ for $(s-t)(a-r)$. To the right of $M_{s}$ occur the elements $D+M_{t},(t=0, \cdots, s-1)$, all of 
which are equal to $M_{s}$ by (1). Since $1 \leqq s-t \leqq Q$, it follows that $D>0$ and, by (2), $D \leqq a-R<a$. Hence the weight of $D+M_{t}$ is $D+m+t q+k$, which will exceed the weight $m+s q+k$ of $M_{s}$ if $D>(s-t) q$, and hence if $a-r>q$, which is true by (10).

To the left of $M_{t}$ occur the elements

$$
a-D+(m+s q+s-1) a+(k-s) b\left(=M_{s}-D\right)
$$

for $s=t+1, \cdots, Q$. We saw that $D \leqq a-R, D>0$. Hence $R \leqq a-D<a$. The weight of the displayed number is $a-D+m$ $+s q-1+k$. This will exceed the weight $m+t q+k$ of $M_{t}$ if $a-D-1+(s-t) q>0$. But its first part is $\geqq 0$ and second part is $>0$.

THEOREM 7. In every row of tablette $T$ the least weight is that of the diagonal element $M_{s}$.

We annex columns to the left of $T$ exactly as for $n=8$. Write

$$
F_{h i}=(m+h Q q+h Q+i q+i-h) a+(k-h Q-i) b .
$$

Since $(Q q+Q+q) a=a-r-R+(Q+1) b$ follows by eliminating $b$ by (1) and applying (2), we see by cancellation that

$$
F_{h i}=a-r-R+F_{(h-1),(i-1)} .
$$

The weight of $F_{h i}$ is $m+k+h Q q+i q-h$. This exceeds the weight of the second member of (12) if and only if

$$
Q q+q-1+R>a-r .
$$

Hence if (13) holds, $F_{h i}$ is not a minimum decomposition when $h>0, i>0$. Also $F_{0 i}=M_{i}$, which is in $T$ for $i=0, \cdots, Q$. Hence for new columns we have $h \geqq 1, i \geqq 1$. Since $F_{h i}$ is then not a minimum decomposition, the same is evidently true of all $j+F_{h i}$. The number at the top of the column containing $F_{h i}$ is the sum of an integer $\geqq 1$ and the function obtained from $F_{h i}$ by subtracting unity from the coefficient of $a$. Hence the entries above $F_{h i}$ are not minimum decompositions.

Theorem 8. In case (13), all minimum decompositions of numbers $<$ c occur in $T$.

Condition (13) holds if $n=2,3,5,6,8-12,14,15$, but for no further $n<36$. For a value of $n$, other than these eleven, it may happen that some number smaller than $B_{k}$ suffices, etc., so that 
our theory is only a first approximation. But a more refined and much more elaborate theory would add no new information about the ideal $I$.

The numbers (6) were obtained as the weights of $j+M_{s}$ for $j=a-r-1, s=Q-1$, and of $i+M_{Q}$ for $i=R-1$, each with $m=q-1$, namely,

(14) $a-r-1+(Q q+Q-2) a+(k-Q+1) b$, of weight $B_{k}$;

(15) $R-1+(q-1+Q q+Q) a+(k-Q) b$, of weight $D_{k}$.

The proof leading to Theorem 2 evidently shows also that if $a>q+r+R$, then $B_{k}-1$ suffices from $k b$ to (14), exclusive, while if $a<q+r+R$, then $D_{k}-1$ suffices from $k b$ to (15), exclusive. Evidently $B_{k-1}=B_{k}-1$.

Since the expectation is that a smaller number suffices after $c$ than before $c$, we shall choose $k$ to be the largest integer for which (14), or (15), is $<c$. In the preceding paragraph we replace $k$ by $k+1$ and conclude that $B_{k}$ or $D_{k}$ suffices to $c$.

The number (4) was obtained as the weight of $i+M_{k}$ for $m=q-1$ :

(16) $a-1-k(a-r)+(q-1+k q+k) a$, of weight $C_{k}$,

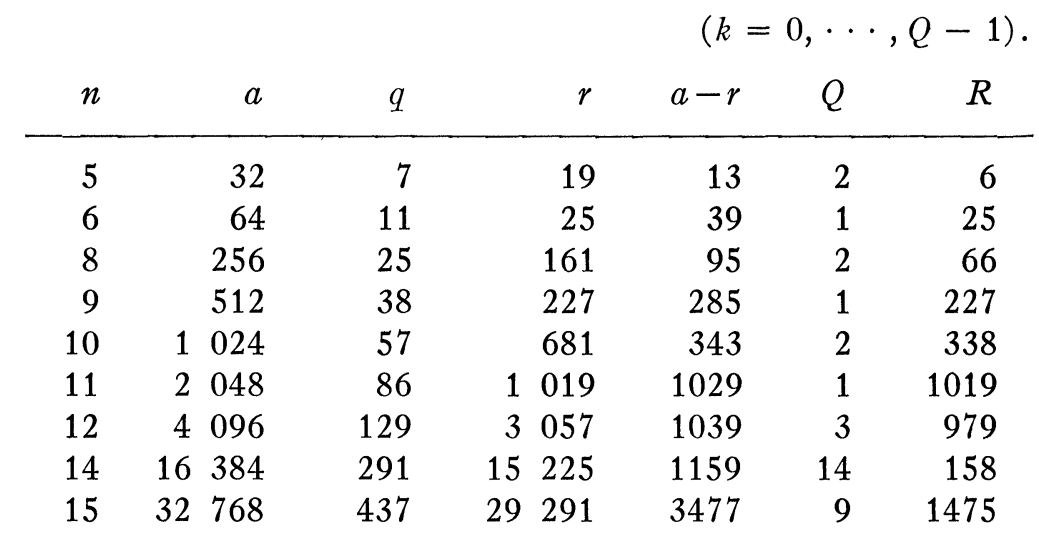

Case $n=8$. Thus $a>q+r+R=252 ;(14)$ is $94+50 a+(k-1) b$. Since $c=248+50 a+8 b$, the largest $k$ is 9 . Theorem 5 states that $C_{0}=279$ suffices for all positive integers $<68 b=446148>5^{8}$, $C_{1}=210$ suffices for all $\geqq b$ and $<68 b$, and $B_{9}=152$ suffices for all $\geqq 2 b$ and $<c$. That no fewer suffice to $c$ follows from Theorems 
7 and 8. Expressed otherwise, only the following peaks occur from 0 to $c$ :

peak 279 at $255+24 a$, peak 210 at $160+50 a$, peak 152 at $94+50 a+8 b$.

Case $n=5$. Peaks $C_{0}=37$ at $31+6 a=223, C_{1}=32$ at $18+14 a$ $=466, B_{3}=28$ at $12+14 a+2 b=946, D_{3}=28$ at $5+22 a+b=952$. Case $n=6$. Peaks $C_{0}=73$ at $63+10 a, B_{4}=52$ at $38+10 a+4 b$. Case $n=9$. Peaks $C_{0}=548$ at $511+37 a, B_{12}=333$ at $284+37 a$ $+12 b$.

Case $n=10$. Peaks $C_{0}=1079, C_{1}=794, D_{16}=523$.

Case $n=11$. Peaks $C_{0}=2132$ at $2047+85 a, D_{22}=1211$ at $1018+172 a+21 b$.

Case $n=12$. Peaks $C_{0}=4223, C_{1}=3314, C_{2}=2405, D_{30}=1523$.

Case $n=14$. Peaks $C_{0}=16673, \cdots, C_{k}=C_{k-1}-867, \cdots$, $C_{13}=5402, B_{14}=5245$.

Case $n=15$. Peaks $C_{0}=33203, \cdots, C_{k}=C_{k-1}-3039, \cdots$, $C_{8}=8891, B_{73}=7481$.

3. Formulas for Ascent. Theorem 9. Let a polynomial $f(x)$ take integral values $\geqq 0$ for every integer $x \geqq 0$ and let

$$
f(x+1)-f(x)
$$

increase with $x$. Make the hypothesis $(H)$ that every integer $i$ for which $l<i \leqq g+f(0)$ is a sum of $k-1$ values of $f(x)$ for integers $x \geqq 0$. Let $m$ be an integer (preferably the maximum one) such that

$$
f(m+1)-f(m)<g-l .
$$

Then every integer I for which $l+f(0)<I \leqq g+f(m+1)$ is a sum of $k$ values of $f(x)$ for integers $x \geqq 0$.

By (1) and (2), we get

$$
f(j+1)-f(j)<g-l, \quad(j=0,1, \cdots, m) .
$$

For a fixed $j$ consider an integer $I$ for which

$$
g+f(j)<I \leqq g+f(j+1) .
$$

Then $g \geqq I-f(j+1)>g+f(j)-f(j+1)>l$ by (4) and (3). Hence, by $(H), i=I-f(j+1)$ is a sum of $k-1$ values of $f(x)$, whence $I$ is a sum of $k$ values. Apply the latter result for $j=0, \cdots, m$ in turn, and note that each of the $m+1$ intervals (4) ends just where the next begins. Hence every integer which exceeds 
$g+f(0)$ and is $\leqq g+f(m+1)$ is a sum of $k$ values. By $(H)$, those from $l$ to $g$ are sums of $k-1$ values. Employ the further value $f(0)$. Hence all from $l+f(0)$ to $g+f(0)$ are sums of $k$ values.

The case $f(x)=x^{n}$ of Theorem 9 may be stated as follows.

THEOREM 10. If every integer $>l$ and $\leqq g$ is a sum of $k-1$ integral nth powers $\geqq 0$, and if $m$ is an integer (preferably the maximum one) for which*

$$
(m+1)^{n}-m^{n}<g-l,
$$

then every integer $>l$ and $\leqq g+(m+1)^{n}$ is a sum of $k$ integral $n$th powers $\geqq 0$.

There are several results $\dagger$ analogous to the following lemma.

LеммA. If $n$ is a positive integer, $l \geqq 0, s \geqq l+1$, there exists a positive integer $i$ such that

$$
l \leqq s-i^{n}<l+n s^{(n-1) / n} .
$$

This implies the following result.

Theorem 11. Let $L>0$ and let $l$ be an integer $\geqq 0$. Write $\sigma=(L / n)^{n /(n-1)}$. If $\sigma \geqq l$ and if all integers between $l$ and $l+L$, inclusive, are sums of $k$ integral $n$th powers $\geqq 0$, then every integer $s$ between $l$ and $\sigma$, inclusive, is a sum of $k+1$ integral $n$th powers $\geqq 0$.

By hypothesis, $l$ is a sum of $k$ and hence of $k+1$ integral $n$th powers. Hence let $s \geqq l+1$. Thus (6) holds for a positive integer $i$. For an $s$ in Theorem $11, s \leqq \sigma$. Hence the final number in (6) is

$$
\leqq l+n \sigma^{(n-1) / n}=l+L .
$$

By the hypothesis in Theorem $11, s-i^{n}$ is therefore a sum of $k$ $n$th powers, whence $s$ is a sum of $k+1$.

Write $L_{0}=l+L, \quad \nu=\left(1-l / L_{0}\right) / n$. Then Theorem 11 with $\sigma=L_{1}$ is equivalent to the following statement.

Let $l$ be an integer $\geqq 0$. Let $L_{0}>l, L_{1}=\left(\nu L_{0}\right)^{n /(n-1)}$. If $L_{1} \geqq L_{0}$ and if all integers between $l$ and $L_{0}$ inclusive are sums of $k n$th powers, then all integers between $l$ and $L_{1}$ inclusive are sums of $k+1 n$th powers.

* Since $x^{n}-(x-1)^{n}<n x^{n-1}$, (5) holds if $m+1$ is $(g-l) / n$ raised to the power $1 /(n-1)$.

$\dagger$ Dickson, American Journal of Mathematics, vol.49 (1927), p. 242; Lemma $2, t=1$. 
Then all between $l$ and $L_{2}=\left(\nu L_{1}\right)^{n /(n-1)}$ are sums of $k+2$ integral $n$th powers since

$$
L_{2} / L_{1}=\left(L_{1} / L_{0}\right)^{n /(n-1)} \geqq 1, L_{2} \geqq L_{1},
$$

and likewise all between $l$ and

$$
L_{t+1}=\left(\nu L_{t}\right)^{n /(n-1)}
$$

are sums of $k+t+1$ powers. The following formula

$$
\log L_{t}=\left(\frac{n}{n-1}\right)^{t}\left(\log L_{0}+n \log \nu\right)-n \log \nu
$$

follows readily from (7) by induction on $t$. Evidently (8) holds if $t=0$.

Theorem 12. Let $l$ be an integer $\geqq 0$. Let

$$
\nu=\left(1-l / L_{0}\right) / n, \quad L_{0}>l, \quad\left(\nu L_{0}\right)^{n /(n-1)} \geqq L_{0} .
$$

Compute $L_{t}$ by (8). If all integers between $l$ and $L_{0}$ inclusive are sums of $k$ integral $n$th powers $\geqq 0$, then all integers between $l$ and $L_{t}$ inclusive are sums of $k+t$ integral $n t h$ powers $\geqq 0$.

4. New Universal Theorems for Fifth Powers. A table giving a minimum decomposition of each integer $\leqq 300,000$ into fifth powers is in press by the British Association for the Advancement of Science. By the table and deductions from it, we find that all numbers from 98604 (the last peak 17) to 191,263 are sums of 16 fifth powers; all from 191,263 (the last peak 16) to 470,348 are sums of 15 ; all from 470,348 (the last peak 15) to 786,159 (the last peak 14) are sums of 14 ; all on to 839,000 are sums of 13 .

We shall make no use of the preceding facts, but rely on the following new tables. A table was made from $A=839,000$ to 929,000 and used to prove that all numbers from $A$ to $B=1,466,800$ are sums of 13 fifth powers. Another table from $B$ to $1,600,000$ showed that 12 powers suffice except for seven numbers which seem to require 13 . It was used to prove that 13 powers suffice from $B$ to $C=3,470,000$. A final table from $C$ to $D=3,600,000$ showed that 11 powers suffice except for 13 numbers which seem to require 12 . It was used to prove that 12 powers suffice from $C$ to $D+18^{5}=5,489,568$. By three applica- 
tions of Theorem 10 with $m=24,40,70$, we find that 15 powers suffice from $A$ to $L_{0}=1,934,501,745$. We apply Theorem 12 with $t=39$ and find that all positive integers $\leqq N$ are sums of $15+39=54$ fifth powers if $\log N=34861$. But James has proved that every integer $>C$ is a sum of 54 if $\log C=34251$.

TheOREM 13.* Every positive integer is a sum of 54 fifth powers. The best earlier result was 58 .

5. New Universal Theorems for nth Powers, $n=6-12$. Gelbcke applied the method of Winogradow to simplify the proof by Hardy and Littlewood of their best result. $\dagger$ James has determined a constant $C$ for which all the inequalities in that paper hold. Every integer $>C$ is a sum of $s n$th powers if $\log _{e} C=20 n^{3} 2^{\eta}$. To determine $\eta$, let $d$ be the largest integer $\leqq \log _{e}(n-1) / \log 2$. Define $\zeta_{k}$ by (2) of $\$ 7$. Write

$$
\begin{aligned}
& D=(d+2)(n-1)-2^{d+1}+0.1, H=(n-2) 2^{n-2}+n, \\
& B=4+\zeta_{k}, R=\left\{1+\left(\frac{n-1}{n}\right)^{B-2}\right\} n 2^{n-2}-D(B+2) .
\end{aligned}
$$

Then

For $n=6,7,8,12, \eta$ is

$$
\eta=\frac{D s+R}{s-H-B-2} .
$$

$$
\begin{aligned}
& \frac{12.1 s-114.3}{s-88}, \frac{16.1 s-186.9}{s-193}, \\
& \frac{20.1 s-162}{s-426}, \quad \frac{39.1 s+9055.4}{s-10335} .
\end{aligned}
$$

R. C. Shook has lately completed extensive tables of minimum decompositions into sixth powers. By them he proved that 23 sixth powers suffice from $2,120,044$ to $2,426,148$. By Theorems 10 and 12 , he found that all integers $\leqq N$ are sums of 160 sixth powers if $\log \log N=11.012$. But $\log \log C=10.890$.

THEOREM 14. Every positive integer is a sum of 160 sixth powers. $\ddagger$

* The earlier discussion in this Bulletin, vol. 37 (1931) p. 551, is incomplete since it used the erroneous $\log C=446$.

$\dagger$ Mathematische Annalen, vol. 105 (1931), pp. 637-652.

$\ddagger$ James had obtained 183. The earlier limit was 478 . 
For $n=7$, the first five peaks are 143 at $127+16 a, 133$ at $116+16 a+b, 123$ at $105+16 a+2 b, 113$ at $94+16 a+3 b=8703$, and 105 at $83+16 a+6 b=15253$. In the short stretch on to $c=4^{7}=16384$ occur two peaks 97 . From 16300 to 22038 the largest minimum, 75 , is that of $10+59 a+6 b$. Using only the last result, we find by Theorem 10 that 90 seventh powers suffice to $18,499,724$. Then by Theorem 11,320 suffice to $n$, where $\log \log n=15.539$. But $\log \log C=15.243$.

TheOREM 15. Every integer is a sum of 320 seventh powers.

I have reduced 320 to 259 . The limit by algebra was 3806 .

For $n=8$, we found the three peaks $279,210,152$ to $c$. The next peaks are 149 at $65+76 a+7 b+c, 137$ at $52+76 a+7 b+2 c$, 122 at $52+50 a+18 b+2 c$, and 119 at $l=393,095$. We verified that 111 eighth powers suffice from $l$ to $g=1,683,200$. Thus $6^{8}-5^{8}<g-l$. Hence in Theorem 10 the maximum $m$ is 5 , whence 112 powers suffice from $l$ to $g+6^{8}=3,362,816$. By repeated use of Theorem 10, it was found that 129 eighth powers suffice from $3 c=196,608$ to $2,235,617 \times 10^{9}$. By Theorem 12 with $t=446$, we find that $129+446=575$ powers suffice to $L$, where $\log \log L=26.777$. But $\log \log C=26.677$.

THEOREM 16.* Every positive integer is a sum of 575 eighth powers.

For $n=12,1469$ powers suffice from $129 a+30 b=16,471,614$ to $b+c=17,308,657$. By Theorem 10 , we find that 1560 powers suffice from $3 b$ to $5,497,325 \times 10^{21}$. By Theorem 12 with $t=9151$, we find that 10711 powers suffice to $L$ if $\log \log L=346.995$. But $\log \log C=346.722$.

THEOREM 17. Every integer is a sum of 10711 twelfth powers.

Kempner $\dagger$ expressed $t\left(x^{2}+y^{2}+z^{2}\right)^{6}$ as a sum of $r 12$ th powers, where $t=12 ! / 16$. I find that $r=13,013,280$ and conclude that every integer is a sum of $k=4223+478 r$ twelf th powers, where $k=6 \frac{1}{4}$ billion. There is no other result by algebra. Starting with very small intervals, James proved this theorem.

* Using merely the fact that 120 suffice from $A=65449$ to $A+a$, inclusive, James verified that every integer is a sum of 595 eighth powers. The earlier limit was 31,353 .

$\dagger$ Mathematische Annalen, vol. 72 (1912), p. 398. See [18] below. 
THEOREM 18. Every integer is a sum of 1177 ninth powers, and of 2421 tenth powers.

I have reduced 1177 to 981 . The earlier result for tenth powers was 140004.

6. Cubes and Fourth Powers. Our method has succeeded for $n \geqq 5$, but fails for $n=3$. The asymptotic theory yields a constant $C$ of about a million digits beyond which every integer is a sum of nine cubes. All integers between 8043 and 40,000 (the limit of tables) are sums of six cubes. Then by Theorem 10 all integers from 8043 to $1,257,375$ are sums of seven cubes. By two more ascents, we conclude that all integers $\leqq 2 \frac{1}{4}$ billion are sums of nine cubes. While this limit is far below $C$, it yields the value beyond which the algebraic proof shows that all integers are sums of nine cubes.

Consider fourth powers (biquadrates). If $x$ is odd, $x^{2}$ is of the form $8 N+1$, whence $x^{4}$ is of the form $16 m+1$. Thus every biquadrate is $\equiv 0$ or $1(\bmod 16)$, so that no integer $\equiv 15(\bmod 16)$ is a sum of 14 or fewer biquadrates. Hence every integer $15+16 m$ requires at least 15 biquadrates.

We can go further and prove that there are infinitely many integers which require at least 16 biquadrates. If a positive integer $K$ is not a sum of fewer than $m$ biquadrates, where $m \leqq 16$, the same is true of $16 K$ and hence of $16^{t} K$, if $t \geqq 1$. For, if $16 K$ were a sum of fewer than $m$ and hence fewer than 16 biquadrates, they must all be even, since any biquadrate is $\equiv 0$.or $1(\bmod 16)$. Removing the factor 16 , we obtain a contradiction to the hypothesis concerning $K$. Evidently if $K$ is a sum of $m$ biquadrates $x_{i}{ }^{4}$, then $16^{t} K$ is the sum of the $\left(2^{t} x_{i}\right)^{4}$. Hence if $K$ has the minimum $m$, where $m \leqq 16$, then $16^{t} K$ has the minimum $m$. Since $31=2^{4}+15$ has the minimum $16,16^{t} \cdot 31$ has the minimum 16 for every $t \geqq 1$.

Bretschneider's table gave a minimum decomposition into biquadrates of each integer $<4100$. Emily Chandler has recently extended the table to 28,561 and proved that all integers between 13,800 and 158,800 are sums of 16 biquadrates. She concludes that all integers $<10^{26}$ are sums of 19 biquadrates. Then by Theorem 12 with $t=16$, all integers $\leqq L$ are sums of 35 biquadrates if $\log \log L=3.37$. By the asymptotic theory all integers $>C$ are sums of 35 if $\log \log C=3.30$. 
TheOREm 19. All positive integers are sums of 35 fourth powers.

The best result by algebra is 37 .

7. Survey of the Literature on Waring's Problem on Powers. That every positive integer is a sum of four integral squares was noted by Bachet in 1621, and a proof by descent claimed by Fermat in 1659, while the first published proof was due to Lagrange in 1770. The same year, Edward Waring [1] conjectured that every (positive) integer is a sum of at most 9 (positive integral) cubes, also is a sum of at most 19 biquadrates, and so on.

Except for squares, the first case of a Waring theorem actually proved is due to Liouville[2]. Every positive integer $P$ is a sum of 53 fourth powers. He used an identity equivalent to, but more complicated than, the identity

$$
\begin{aligned}
6\left(a^{2}+b^{2}+c^{2}+d^{2}\right)^{2}= & (a+b)^{4}+(a-b)^{4}+(a+c)^{4} \\
& +(a-c)^{4}+(a+d)^{4}+(a-d)^{4} \\
& +(b+c)^{4}+(b-c)^{4}+(b+d)^{4} \\
& +(b-d)^{4}+(c+d)^{4}+(c-d)^{4}
\end{aligned}
$$

Every $P$ is of the form $6 q+r$, where $q \geqq 0,0 \leqq r \leqq 5$, whence $r$ is a sum of 5 fourth powers each 0 or 1 . Also, $q$ is a sum of four squares $m_{i}{ }^{2}$. For each $i, m_{i}=a^{2}+b^{2}+c^{2}+d^{2}$, and (1) shows that $6 m_{i}^{2}$ is a sum of 12 fourth powers. Hence $6 q$ is a sum of $4 \times 12$, whence $P$ is a sum of $48+5$ fourth powers.

This limit 53 was reduced to 41 by Lucas in 1878 , to 39 by Fleck in 1906 , to 38 by Landau in 1907, and finally to 37 by Wieferich in 1909.

In 1895, Maillet [3] proved that every positive integer $P$ is a sum of 21 cubes $\geqq 0$, at least 5 of which are 0 or 1 , while all sufficiently large $P$ are sums of 17 .

In 1909, Hilbert [4] proved that every positive integer $P$ is a sum of $N_{m}$ integral $m$ th powers $\geqq 0$, where $N_{m}$ is a finite number (not determined) which depends upon $m$ but not on $P$. By use of a multiple integral, he first proved the existence (conjectured by Hurwitz [5]) of an identity which expresses $\left(x^{2}+\cdots+x_{5}^{2}\right)^{m}$ as a sum of $2 m$ th powers of linear functions 
of $x_{1}, \cdots, x_{5}$ with integral coefficients, each power being multiplied by a positive rational number.

In addition to (1), such an identity had been given by Fleck [6] to obtain $N_{6} \leqq 184 N_{3}+59$; by Hurwitz [5] to get $N_{8} \leqq 36,119$; by Schur [7] to show that $N_{10}$ is finite [140004].

Wieferich [8] proved that every integer is a sum of nine cubes.

By use of the analytic theory of primes, Landau [9] proved that all sufficiently large integers are sums of eight cubes $\geqq 0$.

Wieferich [10] proved that $N_{5} \leqq 59, N_{7} \leqq 3806$.

Hilbert's proof has been materially simplified by Hausdorff [11] and Stridsberg [12]. An exposition of the resulting proof is due to Oppenheim [13]. By altering Stridsberg's proof at the point where he had used integrals, Remak [14], Frobenius [15], and Stridsberg [16] reduced the proof of Waring's theorem to algebraic processes.

There exist infinitely many positive integers which are not sums of $n$ or fewer positive $n$th powers (Hurwitz [5] and Maillet [17]). This follows from $S=1$, where $S$ is the superior limit for $k=\infty$ of $C / k, C$ being the number of positive integers $\leqq k$ which are sums of $n$ or fewer positive $n$th powers.

Kempner [18] proved that $S<1 / n$ ! and concluded that there is an infinitude of positive integers of each of the forms $9 l$, $9 l+1, \cdots, 9 l+8$, such that each is not a sum of fewer than four positive cubes. Also there is an infinitude of positive integers each not a sum of fewer than nine sixth powers, and an infinitude each not a sum of fewer than $2^{q+2}$ powers with the exponent $2^{q}$, for $q>1$.

Kempner used a new identity to reduce Fleck's [6] limit for $N_{6}$ to 970 . While Hurwitz [5] expressed the numbers $<5040$ as sums of units, Kempner noted that they are sums of at most 273 eighth powers (of 1 or 2) and so reduced the limit for $N_{8}$ by $5039-273$. He gave identities which yield limits for $N_{12}$ and $N_{14}$ in terms of $N_{6}$ and $N_{7}$, respectively. (See below Theorem 17.)

Baer [19] reduced the limit for $N_{5}$ to 58 and that for $N_{6}$ to 478. He proved that all large numbers of various linear forms are sums of seven [20] (and others of 8) positive cubes.

Hardy and Ramanujan [21] gave an asymptotic formula (involving the zeta and gamma functions) for the number of 
ways $n$ is a sum of $r$ th powers of positive integers (rearrangements of the same powers not being counted as distinct).

Of prime importance is the theory originated by Hardy and Littlewood, which applies not merely to Waring's problem but also to various other problems in additive number theory, such as the theory of partitions and Goldbach's and related theorems on sums of primes. The general outlines of their method were explained in their initial paper [22] of 1919. Details of proofs are found in their series of memoirs [23] entitled Some Problems of Partitio Numerorum, I-VIII. They obtained asymptotic formulas for the number $r(n, k, s)$ of ways to express a positive integer $n$ as a sum of $s k$ th powers of integers $\geqq 0$. Let $G(k)$ denote the least $s$ such that every sufficiently large integer is a sum of $s k$ th powers. They proved that $G(k) \leqq(k-2) 2^{k-1}+4$, whence it suffices to use 9 cubes, 21 biquadrates, 53 fifth powers, etc. In VI, they proved that, if $k \geqq 4, G(k) \leqq(k / 2-1) 2^{k-1}$ $+k+5+\zeta_{k}$, where

$$
\zeta_{k}=\left[\frac{(k-2) \log 2-\log k+\log (k-2)}{\log k-\log (k-1)}\right] ;
$$

here $[y]$ denotes the greatest integer $\leqq y$. Hence all large numbers are sums of 19 biquadrates, 41 fifth powers, 87 sixth powers, 193 seventh powers, 425 eighth powers, 949 ninth powers, 2113 tenth powers, etc.

Material simplifications in the proofs have been made by Landau [24], Weyl [25], and Gelbcke [26], who applied the method of Winogradow to prove the results in VI.

In II, the question is raised whether or not the asymptotic result for $r(n, 4,21)$ holds true also for $r(n, 4, s), s>21$. This was answered affirmatively by Ostrowski [27], also for $r(n, k, s)$.

Hardy [28] discussed the number of representations as a sum of $n$ cubes.

Western [29] made extensive calculations to find the least $G$ such that all sufficiently large integers are sums of $G$ integral cubes $\geqq 0$. The work of Hardy and Littlewood makes it highly plausible that $G$ is 4 or 5 . An examination of 254,000 numbers made the following inferences appear probable.

There are only a finite number of integers not a sum of five cubes, and the greatest of them is $<2 \cdot 10^{6}$ and is $\equiv \pm 4(\bmod 9)$. 
$G$ is 4 , and the greatest integer not a sum of 4 cubes lies between $10^{12}$ and $10^{14}$.

Landau [30] wrote $N_{k}(x)$ for the number of integers $n \leqq x$ which are sums of two $k$ th powers $\geqq 0$. If $k$ is even, the inferior limit for $x=\infty$ of $x^{-2 a} N_{k}(x) \log x$ is positive. This implies a similar result on the number of integers $n \leqq x$ which are sums of $s k$ th powers.

Chowla [31] proved that every large integer $N$ is the sum of the cubes of eight integers each $>(1 / 9) N^{1 / 3}$; every large $N$ is a sum of eight cubes in at least $g N^{2 / 9}$ ways, where $g$ is a positive constant.

G. K. Stanley [32] proved that every large integer is a sum of two squares and four cubes $\geqq 0$, also is a sum of one square and six cubes $\geqq 0$.

R. D. James [33] has recently improved considerably the best earlier result for the case of odd powers. All sufficiently large integers are sums of $35 \mathrm{fifth}$ powers (in place of the earlier 41 ). If $k$ is odd and $\geqq 7$, all sufficiently large integers are sums of

$$
(k-3) 2^{k-2}+k+9+\zeta k
$$

$k$ th powers, where $\zeta_{k}$ is given by (2). This gives 164 for $k=7$ (instead of the earlier 193), and 824 for $k=9$ (in place of 949).

8. Sums of Powers with Coefficients. The writer [34] proved that every integer $\geqq 0$ is a sum of 17 biquadrates and the doubles of ten biquadrates. Also is a sum of $r$ sixth powers and the products of $s$ sixth powers by 8 , where either $r=115$, $s=108$, or $r=178, s=99$. He proves other similar theorems. Elsewhere he [35] wrote $C_{n}$ for a sum of $n$ cubes and proved that $C_{8}+t x^{3}$ is universal (represents all integers $\geqq 0$ ) if $1 \leqq t \leqq 23$, $t \neq 20$, but not if $t>23$. Also $C_{7}+2 x^{3}+t y^{3}$ is universal if $1 \leqq t \leqq 34$, except when $t=10,15,20,25,30$. Also, $C_{7}+3 x^{3}+t y^{3}$ is universal if $1 \leqq t \leqq 9, t \neq 5$. If $N$ is a positive integer prime to 6 , every integer $\geqq 23^{3} N$ is represented by $6\left(x^{2}+y^{2}+z^{2}\right)+N w^{3}$. For $l=1,2,3,4$, or $5, C_{7}+l x^{3}$ represents all sufficiently large integers.

K. C. Yang [36] obtained limits beyond which every integer is represented by $C_{7}+r x^{3}$ for $r=3,5,7,8$. Every odd integer $\geqq k=23 \cdot 10^{14}$ is represented by $C_{7}+r x^{3}$ for $r=2,4$, or 6 . Every integer $\geqq k$ which is double an odd is represented by $C_{7}+2 x^{3}$.

R. E. Huston [37] employed $s=(k-2) 2^{k-1}+5$, as in the 
first Hardy-Littlewood theory, $f=a_{1} x_{1}{ }^{k}+\cdots+a_{s} x_{s}{ }^{k}$, and proved that all sufficiently large integers are represented by $f$ with each $x_{i}$ an integer $\geqq 0$, if for every integer $n$ there exists a solution of $f \equiv n\left(\bmod p^{\gamma}\right)$ when $p$ is a prime not dividing both $a_{i}$ and $x_{i}$ for $i=1, \cdots, s$, and $\gamma=\theta+1$ if $p>2, \gamma=\theta+2$ if $p=2$, where $p^{\theta}$ is the highest power of $p$ dividing $k$. In various general cases, the congruence was proved to have such a solution. This is true for example if $k=3, a_{1}=a_{2}=1, a_{3}=2,3,4$ or 5 , with $a_{4}, \cdots, a_{9}$ arbitrary; also for $k=4$, if five coefficients are $1,1,2,4,8$, and the remaining 16 are arbitrary.

9. Remarkable Empirical Generalizations of Waring's Theorem. Let $\left(a_{1}, \cdots, a_{r}\right)_{n}$ denote the form $a_{1} x_{1}{ }^{n}+\cdots+a_{r} x_{r}{ }^{n}$, in which the $a$ 's are all positive integers, and call $r$ its order, and $a_{1}+\cdots+a_{r}$ its weight. In $\$ 2$, we discussed the ideal limit $I$ for a universal theorem on $n$th powers. Since $a x^{n}$ is a sum of $a$ $n$th powers, $I$ is the minimum weight of a form which represents all positive integers. We shall here restrict our attention to forms of weight $I$.

If a form of weight $I$ represents all positive integers, its order exceeds 4 if $n=3,6$ if $n=4,7$ if $n=5,9$ if $n=6$, and 10 if $n=7$. But there exist forms of one higher order which represent all integers up to a high limit.

Let $f$ be a form which represents $k$, and let $a_{1}=s+t$. The form $g=\left(s, t, a_{2}, \cdots, a_{r}\right)_{n}$ shall be said to be derived from $f$ by the partition of $a_{1}$ into $s+t$. If we give to the first two variables in $g$ the same value $x_{1}$ that was employed in $f=k$, we see that also $g$ represents $k$. Hence any form derived from $f$ by partition represents every integer which can be represented by $f$ (and usually represents further integers).

It may be shown [38] in a few lines that, if $n=3$, the only forms of weight 9 are (11223), together with all forms

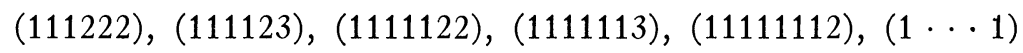

derived from it by partition. Hence if (11223) represents all integers $<k$, the same is true of the remaining six forms. This was verified in the article just cited for $k=1400$. The fact that (11223) represents all integers $<40,000$ was proved in 1929 , 
but is here first published. First, we* added each sum of two cubes to each double of a sum of two cubes to obtain a list of all integers $<40,000$ which are represented by (1122). From each number not in that table we subtracted the triples of small cubes until we found a difference which is not in the table.

Another important result was proved: every integer $>9580$ and $\leqq 40,000$ is a sum of four cubes and the double of a cube. Since this is evidently true for all integers in our table of values of (1122), it sufficed to subtract doubles of small cubes from the numbers not in our table until we reached a difference which is a sum of four or fewer cubes. As a corollary, all integers between 9580 and 40,000 are sums of six cubes. This fact is an essential part of the proof that all integers are sums of nine cubes. That fact had been deduced in 1903 by von Sterneck [39], whose table was made by the successive additions of cubes, until sums of six were found. Any error by that method would introduce extremely many later errors. Our method avoided such a pyramiding of errors, and at any event gave an independent proof.

By means of our table for (1122), I verified also that each of the forms (11224), (11122), and (11222) represents all integers $<40,000$. Hence all the theorems in the Monthly hold to 40,000 .

We shall not quote $[40]$ the interesting empirical theorems for $n=4$.

For $n=5$, the order exceeds 7 . The only two forms of order 8 which represent every integer $\leqq 7007$ are

$$
(1123468,12), \quad(1123457,14) \text {. }
$$

Next, $(1123469,11)$ and $(1123458,13)$ represent all integers $\leqq 7007$ except 1931 and 3137 , respectively. By partitioning a single coefficient of these four forms, we obtain 58 distinct forms of order 9 which represent all integers $\leqq 7007$. Consider $(11 \cdots, 10,10)$ where the suppressed coefficients are 12345 , 22245,22335 , or 22344 ; the first two represent all integers $<3127$, and the last two all $\leqq 2000$.

For $n=6, \mathrm{R}$. C. Shook proved that the order exceeds 9, and obtained 22 forms of order 10 which represent all integers

* Half of the work was done at Chicago, mostly by assistants, and half by J. S. Turner of Ames, Iowa. 
$\leqq 4^{6}=4,096$. They include $(112457,10, x, y, 17)$ for $x, y=10$, $16 ; 11,15 ; 12,14 ; 13,13$.

For $n=7, \mathrm{~K}$. C. Yang proved that the order exceeds 10, and verified that $(11234579,10,15,26,60)$ of order 12 represents all integers $\leqq 16384$. M. E. Mauch obtained various general results for $n=7$ and verified that $(1123469,13,17,41$, 46) of order 11 represents all integers $\leqq 22,000$.

10. Sums of Values of a Polynomial. The early Greeks defined polygonal numbers. For example, the triangular numbers 1, $1+2,1+2+3, \cdots$ count the number of touching equal spheres arranged in the form of a triangle. The $x$ th polygonal number of order $m+2$ (or $m+2$ sides) is

$$
p_{m+2}(x)=x+m\left(x^{2}-x\right) / 2,
$$

whence the $x$ th triangular number is $p_{3}(x)=x(x+1) / 2$. In 1636 , Fermat [41] stated that he was the first to discover the beautiful theorem that every integer $\geqq 0$ is a sum of 3 triangular numbers $\geqq 0, \cdots$, and is a sum of $m$ polygonal numbers $\geqq 0$ of order $m$.

Waring [42] conjectured that every positive integer $P$ "of the proper form" is a sum of a finite number of values of a polynomial $f(x)$, where $P$ is a multiple of 3 if $f(x)=3 x^{4}+6 x^{3}+24$.

Gauss [43] proved that every integer $n=8 m+3$ is a sum of three odd squares $\left(2 a_{i}+1\right)^{2}$, whence $m=\sum\left(a_{i}{ }^{2}+a_{i}\right) / 2$, so that every integer $m \geqq 0$ is a sum of three triangular numbers. The number of ways $m$ can be so decomposed depends in a definite manner on the prime factors of $n$ and the number of classes of binary quadratic forms of determinant $-n$.

Barlow [44] noted that $N$ is a sum of $m+2$ polygonal numbers (1) for $x=x_{1}, \cdots, x_{m+2}$ if and only if

$$
8 m N+(m+2)(m-2)^{2}=\sum_{i=1}^{m+2}\left(2 m x_{i}-m+2\right)^{2} .
$$

Cauchy [45] was the first to publish a proof of Fermat's assertion that every integer $A \geqq 0$ is a sum of $m+2$ polygonal $p_{m+2}$, all but four of which may be taken to be 0 or 1 . In the simplified proof by Legendre [46], the case $m=3$ is not presupposed, as was done by Cauchy. Moreover, Legendre proved that every integer $>28(m-2)^{3}$ is a sum of four $p_{m}$ if $m$ is odd; 
while, for $m$ even, every integer $>7(m-2)^{3}$ is a sum of five $p_{m}$, one of which is 0 or 1 .

Liouville [47] denoted triangular numbers by $\Delta$ 's and proved that

$\Delta+\Delta^{\prime}+c \Delta^{\prime \prime},(c=1,2,4,5), \quad \Delta+2 \Delta^{\prime}+d \Delta^{\prime \prime},(d=2,3,4)$,

represent all integers $\geqq 0$, while no further linear combinations of three $\Delta$ 's with positive integral coefficients represent all integers.

S. Réalis [48] proved that every integer is a sum of four of the numbers $\left(3 z^{2} \pm z\right) / 2$, and also a sum of four of the form $2 z^{2} \pm z$, that is, $p_{5}$ and $p_{6}$ extended to negative arguments. [But three suffice, \$11.]

Maillet [49] proved that if $\alpha$ and $\beta$ are relatively prime odd integers, $\alpha>0$, every integer $A$ exceeding a certain limit (depending on $\alpha, \beta)$ is a sum* of four integers $\geqq 0$ of the form $\left(\alpha x^{2}+\beta x\right) / 2$. Next let $\alpha$ and $\beta$ be both even and let $\alpha / 2$ and $\beta / 2$ be relatively prime. Then every sufficiently large odd integer is a sum of four integers $\left(\alpha x^{2}+\beta x\right) / 2$ if $\alpha / 2+\beta / 2$ is odd.

Let $\alpha$ and $\beta$ be relatively prime odd integers. According as the g. c. d. of $(\alpha+\beta) / 2$ and 6 is $1,2,3$ or 6 , take $\rho=0,1,2,3,4$, or $5 ; \rho=0,2$, or $4 ; \rho=0$ or $3 ; \rho=0$, respectively. Then every sufficiently large integer $\equiv \rho(\bmod 6)$ is a sum of 53 values of $\alpha x^{4} / 2+\beta x^{2} / 2$ for integers $x \geqq 0$. He gave similar theorems when $\alpha$ and $\beta$ are even.

Maillet [50] proved that, if a polynomial $f(x)$ is an integer $\geqq 0$ for every integer $x \geqq \mu$, then every integer exceeding a fixed limit, depending on the coefficients of $f(x)$, is a sum of at most $n$ positive values of $f(x)$ and a limited number of 1 's, where $n=6,12,96$, or 192 , according as the degree of $f$ is $2,3,4$, or 5 , respectively. Every integer $\geqq 19272$ is a sum of at most 12 pyramidal numbers $\left(x^{3}-x\right) / 6$.

Kamke [51] proved the following precise formulation of Waring's conjecture. Let $f(x)$ be a polynomial of degree $n \geqq 2$ having rational coefficients, that of $x^{n}$ being $>0$, such that $f(x)$ is an integer $\geqq 0$ for every integer $x \geqq 0$; then every integer $\geqq 0$ is a sum of a limited number of 1 's and a limited number of values of $f(x)$ for integers $x \geqq 0$. The proof rests on the following

* We can assign an inferior limit to $A$, such that this decomposition can be made in any assigned number of ways. 
interesting theorem on the simultaneous decomposition of $n$ positive integers into powers of the same positive integers. If $n$ is a given integer $\geqq 2$, there exist an integer $N=N(n)>0$, an integer $A>0$, and positive numbers $i_{1}, i_{\nu}, J_{\nu}$ satisfying $0<i_{\nu}$ $<J_{\nu}, \quad(\nu=2, \cdots, n)$, such that for all integers $l_{1}, \cdots, l_{n}$ divisible by $A$, satisfying the inequalities

$$
l_{1}>i_{1}, \quad i_{\nu} l_{1}{ }^{\nu}<l_{\nu}<J_{\nu} l_{1}{ }^{\nu}, \quad(\nu=2, \cdots, n),
$$

the $n$ equations

$$
l_{\nu}=x_{1}^{\nu}+\cdots+x_{N}^{\nu}, \quad(\nu=1, \cdots, n),
$$

are solvable simultaneously in integers $x_{1}, \cdots, x_{N}$.

Kamke [52] proved the following generalization of his preceding result. If $f(x)=a_{1} z^{k}+\cdots+a_{k}$ is an integer for every integer $x$, if $a_{k}>0$, and if $T$ is the g. c. d. of the values of $f(x)$ for all integers $x$, then for all sufficiently large integers $Z, T Z$ is a sum of a limited number of values $\geqq 0$ of $f(x)$. When $a_{k}=0$, it holds if $T$ is the g. c. d. of all values of $f(x)-a_{k}$, and the $T Z$ are replaced by the numbers of a suitably chosen class of residues modulo $T$.

Kamke's [51] existence theorem has been proved by the method of Hardy and Littlewood by Winogradow [53] and Landau [54].

The writer [55] proved that the only quadratic functions of $x$ which are integers $\geqq 0$ for every integer $x \geqq 0$ and which take the values 0 and 1 for certain integers $x \geqq 0$ (necessary for a universal theorem) are the functions derived from the polygonal number (1) by replacing $x$ by $x-k$ or $k-x$, where $k$ is an integer $\geqq 0$. He found the least $l$ such that every integer $\geqq 0$ is a sum of $l$ values of such a function.

Consider any quadratic function $q(x)$ whose value is an integer $\geqq 0$ for every integer $x \geqq 0$. The problem to find the least $e_{s}$ such that every positive integer is a sum of $s$ values of $q(x)$ for integers $x \geqq 0$ and $e_{s}$ terms 0 or 1 has been treated at length [56].

L. W. Griffiths [57] found all functions $a_{1} P_{1}+\cdots+a_{n} P_{n}$ which are universal, that is, represent all integers $\geqq 0$, where the $P$ 's are polygonal numbers of order $m+2$ and the $a$ 's are positive integers whose sum is $\leqq m+2$. She solved [58] the like 
problem when $P$ is $p_{m+2}(1-x)$, and partially the problem when $P$ is the function ( 1$)$ for $x=0, \pm 1, \pm 2, \cdots$.

Every integer is a sum of a square and two triangular numbers, and vice versa [59].

Dickson [60] proved that every sufficiently large integer is a sum of nine values of $x+k\left(x^{3}-x\right) / 6$ for integers $x \geqq 0$, when $k$ is prime to 3 . This is true of all integers if $k$ is 1 or 2 .

James [61] proved that all sufficiently large integers are sums of eight pyramidal numbers $\left(x^{3}-x\right) / 6$.

11. Sums of Values of Two or More Polynomials. For this new problem we have only examples.

Consider (1) and the extended polygonal number $e_{m+2}(x)$ of order $m+2$ which is derived from (1) by replacing $x$ by $-x$. Both $p$ and $e$ are integers $\geqq 0$ for every integer $x \geqq 0$.

By a standard theorem, every positive integer of the form $8 n+3$ is a sum of three squares, each of which is evidently odd. Any odd is of the form $4 x \pm 1$. Hence $8 n+3=\sum(4 x \pm 1)^{2}$ for three integers $x \geqq 0$. Hence $n=\sum\left(2 x^{2} \pm x\right)$. For the upper sign, the summand is $e_{6}(x)$; for the lower sign, it is $p_{6}(x)$. Hence every integer $\geqq 0$ is a sum of three numbers each of which is a value of one or the other of $p_{6}(x), e_{6}(x)$.

Similar theorems hold [62] for every $m$.

From $8 n+3=\sum(2 y+1)^{2}$, we see that every positive integer $n$ is a sum of three triangular numbers $p_{3}(y)=\left(y^{2}+y\right) / 2$. According as $y=2 x$ or $y=2 x-1, p_{3}(y)=e_{6}(x)$ or $p_{6}(x)$. This gives another proof of the theorem in italics. This method is applicable to any polynomial with fractional coefficients. For example, from $p_{5}(y)$ we get the pair $6 x^{2}-x, 6 x^{2}+5 x+1$. Hence every integer is a sum of five values of these two functions for integers $x \geqq 0$. Taking $x$ to be $3 y, 3 y+1,3 y+2$ in turn in the last theorem of $\$ 10$, with $k=1$, we see that every integer is a sum of nine values of

$$
9 y^{3}+2 y, 9 y^{3}+9 y^{2}+5 y+1,9 y^{3}+18 y^{2}+14 y+4
$$

for in tegers $y \geqq 0$.

12. Universal Sums of a Quadratic Form. A binary quadratic form which represents unity is evidently equivalent to $B(x, y)=$ $x^{2}+g x y+h y^{2}, g=0$ or 1 . The case of sums of four or more values of $B$ is trivial since every integer is a sum of four squares. 
The writer [63] proved that every positive integer is a sum of two values of $B(x, y)$ for integers $x \geqq 0, y \geqq 0$ if and only if $h=1,2$, or 3 ; and is a sum of three values of $B$ if and only if $h=1, \cdots, 7$.

Asymptotic expressions have been found [64] for the number of representations of $n$ by $N\left(b_{1}\right)+\cdots+N\left(b_{r}\right)$, where $N\left(b_{i}\right)$ is the norm of an ideal $b_{i}$ of a class $K_{i}$ of ideals of the quadratic field defined by $\left(D_{i}\right)^{1 / 2}$.

\section{BIBLIOGRAPHY}

1. Meditationes Algebraicae, Cambridge, 1770, pp. 204-205; 3d ed., 1782, pp. 349-350.

2. Lectures at the Collège de France; printed in V. A. Lebesgue's Exercices d'Analyse Numérique, Paris, 1859, pp. 112-115.

3. Association Française Avancement des Sciences, vol. 24, II (1895), pp. 242-247.

4. Göttinger Nachrichten, 1909, pp. 17-36; Mathematische Annalen, vol. 67 (1909), pp. 281-300. See Schmidt, ibid., vol. 74 (1913), pp. 271-274.

5. Mathematische Annalen, vol. 65 (1908), pp. 424-427.

6. Mathematische Annalen, vol. 64 (1907), pp. 561-566.

7. Mathematische Annalen, vol. 66 (1909), p. 105.

8. Mathematische Annalen, vol. 66 (1909), pp. 95-101. A case overlooked was treated unsuccessfully by Bachmann and Lejneek, but the gap was finally filled by Kempner [18]. The proof was much simplified by Dickson, Transactions of this Society, vol. 30 (1928), pp. 1-18.

9. Mathematische Annalen, vol. 66 (1909), pp. 102-105; Landau, Handbuch der Lehre von der Verteilung der Primzahlen, vol. I, 1909, pp. 555-559. For numbers prime to 10 (without the theory of primes), Archiv der Mathematik und Physik, (3), vol. 18 (1911), pp. 248-252.

10. Mathematische Annalen, vol. 67 (1909), pp. 61-75.

11. Mathematische Annalen, vol. 67 (1909), pp. 301-305. See Hurwitz, ibid., vol. 73 (1912), pp. 173-176.

12. Arkiv för Matematik, Astronomi, Fysik, vol. 6 (1910-11), No. 32, No. 39. Summary in French in Mathematische Annalen, vol. 72 (1912), pp. 145152.

13. Mathematische Zeitschrift, vol. 31 (1929), pp. 149-150.

14. Mathematische Annalen, vol. 72 (1912), pp. 153-156.

15. Sitzungsberichte Akademie Wissenschaften, Berlin, 1912, pp. 666-670.

16. Arkiv för Matematik, Astronomi, Fysik, vol. 11 (1916), No. 25 ; vol. 13 (1919), No. 25.

17. Bulletin Société Mathématique de France, vol. 36 (1908), pp. 69-77.

18. Über das Waringsche Problem und einige Verallgemeinerungen, Dissertation, Göttingen, 1912. Extract in Mathematische Annalen, vol. 72 (1912), p. 387.

19. Beiträge zum Waringschen Problem, Dissertation, Göttingen, 1913. 
20. Extensions by Post, Dissertation, Halle, 1920, and Arndt, Dissertation, Göttingen, 1925.

21. Proceedings London Mathematical Society, (2), vol. 16 (1917), p. 130.

22. Quarterly Journal of Mathematics, vol. 48 (1919), pp. 272-293. See Hardy, Some Famous Problems of the Theory of Numbers, and in particular Waring's Problem, Oxford, 1920.

23. I. Göttinger Nachrichten, 1920, pp. 33-54.

II. Mathematische Zeitschrift, vol. 9 (1921), pp. 14-27.

III. Acta Mathematica, 1922, pp. 1-70 (sum of primes).

IV. Mathematische Zeitschrift, vol. 12 (1922), pp. 161-188.

V. Proceedings London Mathematical Society, (2), vol. 22 (1923), pp. 46-56 (Goldbach's problem).

VI. Mathematische Zeitschrift, vol. 23 (1925), pp. 1-37.

VII. Proceedings London Mathematical Society, (2), vol. 28 (1927), pp. 518-542.

24. Göttinger Nachrichten, 1921, pp. 88-92; Mathematische Zeitschrift, vol. 12 (1922), pp. 219-247; Proceedings London Mathematical Society, (2), vol. 25 (1926), pp. 484-485. Vorlesungen über Zahlentheorie, I, 1927, pp. 235360 (pp. 276-303 may be replaced by his simpler proof in Mathematische Zeitschrift, vol. 32 (1930), pp. 699-702). See papers [53], [54].

25. Göttinger Nachrichten, 1921, pp. 189-192; 1922.

26. Mathematische Annalen, vol. 105 (1931), pp. 637-652.

27. Mathematische Zeitschrift, vol. 9 (1921), pp. 28-34.

28. British Association Report, vol. 90 (1922), pp. 16-24.

29. Journal London Mathematical Society, vol. 1 (1926), pp. 244-245.

30. Ibid., pp. 72-74. Improved results by Pillai, ibid., vol. 3 (1928), pp. $56-61,83$.

31. Ibid., vol. 5 (1930), pp. 155-158.

32. Ibid., vol. 6 (1931), pp. 194-197.

33. Proceedings London Mathematical Society, 1933-34.

34. American Journal Mathematics, vol. 49 (1927), pp. 241-250.

35. Transactions of this Society, vol. 30 (1928), pp. 1-18.

36. Dissertation, Chicago, 1928.

37. Proceedings London Mathematical Society, 1933-34.

38. Dickson, American Mathematical Monthly, vol. 34 (1927), pp. 177-183.

39. Sitzungsberichte, Akademie der Wissenschaften, Wien, vol. 112, II a (1903), pp. 1627-1666.

40. Dickson, this Bulletin, vol. 33 (1927), pp. 319-325.

41. Oeuvres, vol. I, p. 305 ; vol. II, p. 65 . French translation, III, p. 252, p. 287.

42. Meditationes Algebraicae, 1770, pp. 204-205; 3d ed., 1782, pp. 349-350.

43. Disquisitiones Arithmeticae, 1801, §293; Werke, I, 1863, p. 348.

44. Theory of Numbers, 1811, p. 219.

45. Mémoires Scientifiques Mathématiques et Physiques de l'Institut de France, (1), vol. 14 (1813-15), pp. 177-220; same in Exercices de Mathematiques, vol. I, 1826, pp. 265-296; Oeuvres de Cauchy, (2), vol. VI, pp. 320-353; Journal des Mines, vol. 38 (1815), p. 395. The proof by Pepin, Atti Accademia Pontifica Nuovi Lincei, vol. 48 (1892-93), pp. 119-131, holds 
when $A>110 m$, and was verified by a table for all $A<120 m+16$. The simpler proof by Dickson [56] requires a table only to $48 m+21$.

46. Theorie des Nombres, first supplement, 1816, to the second edition, 1808, pp. 13-27; 3d ed., 1830, vol. I, p. 218; vol. II, p. 340; German translation by Maser, vol. 2, p. 332 .

47. Journal de Mathématiques, (2), vol. 7 (1862), p. 407; vol. 8 (1863), p. 73.

48. Nouvelles Annales Mathématiques, (2), vol. 12 (1873), p. 212; Nouvelle Correspondance Mathématique, vol. 4 (1878), pp. 27-30.

49. Bulletin Société Mathématique de France, vol. 23 (1895), pp. 40-49.

50. Journal de Mathématiques, (5), vol. 2 (1896), pp. 363-380.

51. Mathematische Annalen, vol. 83 (1921), pp. 85-112. In Journal für Mathematik, vol. 152 (1923), pp. 30-32, he evaluated $A$ when we employ only the values $l$ and $n$ of $\nu$.

52. Mathematische Zeitschrift, vol. 15 (1922), pp. 188-194; vol. 19 (1924), p. 263.

53. Recueil Société Mathématique Moscow, vol. 31 (1924), pp. 490-507. Akademia Nauk, Bulletin, 1928, pp. 393-400; pp. 401-414. (In Russian.)

54. Acta Mathematica, vol. 48 (1926), pp. 217-253; Mathematische Zeitschrift, vol. 12 (1922), pp. 219-247; vol. 31 (1929), pp. 149-150, 319-338.

55. This Bulletin, vol. 33 (1927), pp. 713-720; vol. 34 (1928), pp. 63-72, 205-217.

56. Dickson, Journal de Mathématiques, (9), vol. 7 (1898), pp. 328-336; American Journal Mathematics, vol. 50 (1928), pp. 1-48; Pall, ibid., vol. 54 (1932), pp. 66-78; Transactions of this Society, vol. 34 (1932), pp. 98-125; this Bulletin, vol. 37 (1931), pp. 267-270; Quarterly Journal Mathematics, vol. 2 (1931), pp. 136-143.

57. Annals of Mathematics, (2), vol. 31 (1930), pp. 1-12.

58. Abstracts in this Bulletin, vol. 35 (1929), p. 767 ; vol. 36 (1930), p. 806.

59. Nouvelles Annales Mathématiques, (2), vol. 11 (1872), pp. 95-6, 516-519; (2), vol. 12 (1873), p. 217.

60. Transactions of this Society, 1933-34.

61. Mathematische Annalen, 1933.

62. Dickson, Journal de Mathématiques, (9), vol. 7 (1928), p. 327.

63. Journal de Mathématiques, (9), vol. 7 (1898), pp. 319-326.

64. Peterson and Rademacher, Abhandlungen, Mathematisches Seminar Hamburg Universität, vol. 4 (1925), pp. 267-296; vol. 5, pp. 40-44.

The University of Chicago 